\title{
Research on Real-time Intrusion Detection Technology Based on Data Mining
}

\author{
Fan Zhiqin ${ }^{1,}$, Wang Mian ${ }^{2}$ \\ ${ }^{1}$ Jiangxi Modern Polytechnic College, Nanchang 330095, China \\ afanzhiqin@126.com
}

Keywords: Intrusion detection, data mining, ant colony optimization clustering, expert system

\begin{abstract}
As the development of computer network, especially the Interact techniques, network is playing more and more important roles in our daily life as well as work.With nodes of the vast network, more and more sensitive information is being stored and manipulated online, the computer network is more vulnerable to miscellaneously malicious or unauthorized actions, network security is becoming increasingly important. The traditional network security techniques, which are passive defense, such as IA(Identification and Authentication), Firewall and SO on, cannot already satisfy the need of network security.
\end{abstract}

The intrusion detection, which is active defense technique, has become another important means and the second floor defense in network security. Intrusion detection has become a hot point domain of research. Nowadays, IDS based on signatures fails to be generalized to detect new attacks or attacks without known signatures. Because data mining technique can detect new or unknown attack from the large network connected data, it becomes the hot point domain in the research of intrusion detection. This dissertation mainly focus on intrusion detection based on data mining. The aim is to improve the detection rate and decrease t11e false alarm rate. and the main research method is clustering analysis.

The algorithm and model of intrusion detection ale proposed and the corresponding simulation experiments are presented. The basic concepts, principles, classification and development of data mining and intrusion detection technique are introduced, and the application background of the data mining in intrusion detection is analyzed. The development and future of intrusion detection based on data mining are proposed.

\section{Network Security}

A computer network, especially Internet, will be a necessary condition for the operation of the information society in the $2 \mathrm{l}$ century. At the same time, network security is becoming the foundation of a country's normal operation of politics, military, economy and social life. It will be an important embodiment of a country's comprehensive strength. Network security means that the data and hardware of the network system and the data in the system are protected, not damaged, altered or leaked because of accidental or malicious reasons, so that the system can run continuously, reliably and frequently, and the network service is not interrupted. Network security includes two parts: system security and information security. System security mainly refers to the security of network devices, such as hardware, operation system, and application software. Information security mainly refers to the security of information storage and transmission, which is embodied in the integrity, availability, and secrecy of information.

On the computer network security threat mainly comes from the following: Fang Li (1) natural disasters, such as earthquake, fire and lightning; (2) human errors, such as the wrong instruction, error data, delete and modify inappropriate system configuration; (3) computer virus; (4) people drive attack and invasion; (5) due to security vulnerabilities of operating system and application software and various protocols caused by "BUG". In the early stage of network generation, network security is mainly caused by the first and 22 aspects, especially human error. In 1994, the NCSA report showed that about $75 \%$ of the network security problems at that time were caused by human 
errors. But with the development of network technology, the improvement of equipment performance and personnel quality, the network security problems caused by human errors and natural disasters are decreasing year by year.

The network security problems caused by hackers, viruses and various "BUG" have accounted for over $80 \%$, which is related to the openness, universality, and instability of Internet. There are many ways to solve the threat of network security. Now the commonly used traditional network security technologies, such as authentication, encryption, firewalls and so on, and active defense intrusion detection technology has also become the second line of security defense, playing a more important role.

\section{The Necessity of Intrusion Detection}

Given the importance of network security, the security of the network security measure is necessary, technology measure of network security from the confidentiality, integrity, availability, authenticity, practicality, possession of six to consider. Secrecy is to protect the information security requirements of the features can only be used by the approved personnel of computer network and information system is to protect the integrity of computer network and information system in the information safe and effective, a feature not because of other unsafe factors and change the original content, form or flow.

That cannot be modified by the unauthorized third party; availability is the security of computer network and information system resources no matter through what kind of treatment, as long as the need is not available, because the system failure or other causes of failure, loss of resources or hinder the use of resources, it will make a kind of strict time service is not timely response; authenticity is the guarantee of entity information and system characteristics of a credible, mainly refers to the integrity of the information system and the practice The confirmation of body is practicality, which is a characteristic of controlling information dissemination and content within the authorized scope; occupancy is a characteristic of ensuring entity's possession of information. To achieve the above six indicators, the ideal way to deal with an intrusion attempt is to establish a completely secure system. However, practical experience has proved that it is impossible to establish a completely safe system. After studying the current popular operating systems and applications, Miller points out that there can be no defects in the software.

Network intrusion behavior often occurs, although firewalls and other security tools have been widely applied to various network environments, there are still many security risks, especially internal staff abuse, and misuse, firewall and other traditional network security tools, basically do not work, and the intrusion detection system is to solve these problems the effective means and tools. Also, identification of authentication, access control, security mechanisms, and firewalls are not possible. Completely prevent intruders by defects and system configuration error operating system and application to network intrusion system, encryption method itself has some problems, the security system is vulnerable to internal user abuse of privilege attacks, security access control level and user efficiency is inversely proportional to the access control and protection model itself has some problems. There is not enough software testing, software life cycles, large software complexity problem in software engineering. Therefore, the practical way to solve the above problems is to set up a relatively easy implementation of the security system, and establish a corresponding safety assistant system according to a certain security strategy. Intrusion detection technology plays a very important role in the establishment of this system.

\section{Intrusion Detection Technology}

Intrusion detection can be technically divided into two types: misuse detection (Misuse Detection) and anomaly detection (Anomaly Detection). The key idea of misuse detection is to define the intrusion mode library in advance by experts according to the known attack method, and then detect the intrusion behavior by matching the pre-configured intrusion mode with the monitored intrusion. Due to the judgment based on intrusion mode library, the detection accuracy is 
high, and the false alarm is few.

However, the misuse detection needs updating the mode library constantly, and it is unable to detect the limitations of the new unknown attack. Because of the new network attack, it is necessary to update and upgrade IDS regularly. Therefore, the misuse detection is adaptable, maintainable and scalable. The commonly used techniques for misuse detection include conditional probability, model misuse reasoning, state migration analysis, expert system, state transformation analysis, genetic algorithm and so on. Anomaly detection first establishes a normal behavior mode library of system or user.

Then, according to the behavior or resource usage mode of the monitored system or user, compared with the normal mode, it detects the occurrence of intrusion by analyzing the deviation degree between them. Usually some amount of statistics collected from the audit records, for each user (user group) to establish activity description file (Profile), it is the probability of normal behaviors, when the user behavior and user profile differences to a certain extent, which is detected and contrary to normal behavior under the condition of it that the invasion occurred, activity description documents should be based on the need for regular updating. Anomaly detection can detect new or unknown attacks, so it has strong applicability, but because of the user's behavior in the constantly changing, activity description file may not always accurately describe the behavior of users, so there is a high rate of false detection of defects, especially in a large number of users often change or system parameters, network structure, working conditions, the environmental factors. Anomaly detection has become the focus of research in the field of intrusion detection. Anomaly detection is based on feature selection, Bias reasoning, data mining, neural network, immune principle, machine learning, pattern prediction, statistical analysis, intuitive sequence mode and so on.

\section{Research on Intrusion Detection Technology Based on Data Mining}

Intrusion detection technology based on Guan Cong rule. In the network security system, association analysis can be used to find the correlation between the invaders' various intrusion behaviors. Association rule mining is one of the most widely used technologies in data mining, and it is also the first technology used in intrusion detection. There are many association rules algorithms, such as Apriori algorithm, which is used in intrusion detection. Association rules are first used to analyze network traffic data. Subsequently, mining results of association rules are used as input data of post-mining, to extract better results. Intrusion Detection Based on clustering analysis.

Clustering analysis is an unsupervised learning method. It divides some unknown models into several classes. If the distance between eigenvectors is equal within a certain error range, they are considered to be the same type. Based on clustering analysis, the basic idea of intrusion detection algorithm is based on two assumptions: the difference between intrusion and normal mode and the number of normal actions should be far greater than the two conditions of intrusion behavior, so that we can divide data sets into different categories. Intrusion detection and data mining technology from eight to distinguish normal and abnormal behavior to intrusion detection.

Clustering and fuzzy clustering algorithms are commonly used Clustering, self-organizing mapping (Sold) neural network clustering, genetic clustering, etc., clustering methods do not need manual or other classification. There is no need for training. Therefore, new and unknown intrusion types can be found. First, a clustering analysis based intrusion detection technology is proposed. After normalization of data instances and conversion to standard form, the technology adopts standard Euclidean metric, uses single link clustering, identifies and classifications to detect intrusion behavior.

Fusion of data mining algorithms and intrusion detection with other detection techniques. A single data mining algorithm is not effective for intrusion detection, because intrusion detection itself is a complex knowledge mining process. Therefore, we can combine these data mining technologies into their characteristics according to their respective characteristics in the detection process, so that they can cooperate with each other and make good use of their advantages to 
playing a role in their respective environments. For example, sequence analysis can be used. There are frequent intrusion sequences, then classification rules are extracted, cluster analysis is used to cluster security events, and the normal behavior and abnormal behavior are classified. Due to the diversity of attack methods and the variability of detection environment, the use of single detection method has some limitations in intrusion detection, or can not detect unknown intrusions or detection rate is not high enough to achieve effective detection targets. Therefore, the use of a variety of detection techniques for intrusion detection is concerned by researchers, such as joint misuse detection and anomaly detection. At present, a variety of hybrid detection methods have been proposed.

\section{The Development Direction of Intrusion Detection Technology}

IDS has matured along with the development of network technology and related disciplines, but it has not reached the perfect level, and its research will not stop. The trend of its development in the future is mainly manifested in the following aspects: A single security technology is not enough to fully protect the network security. Therefore, the full cooperation of various security technologies and their advantages in their respective fields will maximize the protection of network security. When establishing intrusion detection standard and interface, multi-point analysis and correlation technology can be used to improve the accuracy of detection, and develop an interactive mechanism with other security devices, to build a comprehensive, real-time and dynamic security system. Research on intrusion response technology.

When IDS analyses intrusion or suspicious phenomena, the system needs to take appropriate measures to minimize the loss caused by the invasion. The administrator can be notified by generating event alerts, E.mail, or short messages. With the increasing complexity of the network and the improvement of security requirements, the methods of more real-time and automatic response to intrusion are gradually studied and applied. This kind of intrusion response is roughly divided into three types: system protection, dynamic strategy, and attack confrontation. These three aspects are the protection system against network intrusion category, to reduce the losses for dynamic strategy to improve system security responsibilities, and can not only real-time protection against intrusion system, but also can realize the tracking and anti -intrusion active defense strategy. In a word, intrusion detection technology is a hot topic in current network security research. Its rapid development and potential application prospect need more researchers to participate.

Only when IDS develops at the same time in many aspects of basic theory, can it improve its overall performance in an all-round way. Through the above analysis, data mining intrusion detection was put forward which was developed based on today in many ways has been fruitful, but it is still far from the practical level, has not formed a complete theoretical system, so the research still needs to continue. On this issue, we mainly solve the problems of data mining, such as real-time detection, detection rate, false alarm rate and so on, and enrich the development of the existing rationale in the year to make the intrusion detection system more perfect and put into practical stage.

General intrusion detection based on data mining should be studied in the following aspects: system architecture: The first should be a relatively perfect system structure to guide the design and implementation of intrusion detection system based on data mining; Improve the detection performance of mining algorithms: improve with the degree of data mining and intrusion the research for the detection of intrusion detection, data mining algorithms, to improve the detection rate of intrusion detection, reduce the false detection rate: real-time intrusion detection: the only Data Mining Intrusion Detection for real-time detection based on, in order to make this technology really put into combat, this should be one of the key technology at present study. Integration with other detection technologies: data mining should integrate with other detection technologies, and play their respective advantages, so that we can truly play the advantages of data mining. Therefore, the hybrid detection technology suitable for intrusion detection should be one of the future research directions. 


\section{References}

[1] Kgun,etc. State Transition Analysis: A Rule-based Intrusion Detection Approach [J]. IEEE Transactions on Software Engineering, 2013, 21(3): 181-199.

[2] Kumar, etc. A Software Architecture to Support Misuse Intrusion Detection [C]. Proceeding of the 18th National Information Security Conference, 2005. 194-204.

[3] Lee, etc. Data Mining in Work Flow Environment: Experience in Intrusion Detection[C]. Proceedings of the Conference on Knowledge Discovery and Data Mining (KDD-99), 2009.

[4] Ghosh, etc. A Study in Using Neural Networks for Anomaly and Misuse Detecion[C]. In Proceedings of the Eighth USENIX Security Symposium, 2012.

[5] Warrender, etc. Detecting Intrusions Using System Calls: Alternative Data Models[C]. 1999 IEEE Symposium on Security and Privacy, IEEE Computer Society, 2009. 133-145. 